binding to proteins and regulating cellular metabolism. The aim of this study was to identify a certain lncRNA promoting the progress of advanced colorectal cancer (CRC) with a therapeutic perspective.

Methods We screened out highly expressed lncRNAs using samples from patients with stage IV CRC compared with matched adjacent normal tissues. The proteins interacted with linc00920 was confirmed with RNA pull-down and RNA immunoprecipitation (RIP) assay. The proliferation and metabolic alteration of CRC under linc00920 inhibition were tested in vitro and in vivo.

Results Linc00920 was upregulated in CRC with poor overall survival and inhibition of linc00920 resulted in impaired growth of CRC cell lines. Moreover, knocking down of linc00920 was consistent with a lower level of insulin-like growth factor 2 mRNA-binding protein 2 (IGF2BP2), which is known as a $\mathrm{m}^{6} \mathrm{~A}$ 'reader' and mRNA stabilizer. Linc00920 bound to the ubiquitination sites of IGF2BP2 and prevented its autophagic degradation, maintaining the MYC-mediated glycolysis in CRC. Moreover, inhibition of linc00920 suppressed the proliferation of tumors from patient-derived xenograft (PDX) models.

Conclusions Linc00920-IGF2BP2-MYC axis promotes the progress of $\mathrm{CRC}$ as a promising therapeutic target.

\section{IDDF2019-ABS-0269 OVEREXPRESSION OF PDEF SUPPRESSES CELL AGGRESSIVENESS IN COLORECTAL CANCER}

Jia-ni Chen ${ }^{*}$, Wang-xiong Hu, Shu Zheng. Cancer Institute, the Second Affiliated Hosptial, Zhejiang University School of Medicine, China

\subsection{6/gutjnl-2019-IDDFabstracts.53}

Background Prostate-derived Ets factor (PDEF) belongs to the Ets family of transcription factors. It plays an important role in tumorigenesis and progression of many tumors such as prostate cancer, breast cancer, and gastric cancer. However, its biological function in colorectal cancer (CRC) is still unclear.

Methods A lentivirus vector overexpressing PDEF was constructed and transfected into SW620 cells with lipofectine. In order to knock down the expression of PDEF, PDEF-custom si-RNA was transfected into sw620 cells. The expression level of PDEF was detected by western blot and real time-polymerase chain reaction. The proliferation, invasion, migration and cell cycle of SW620 cells were investigated after transfection. Meanwhile, transfected SW620 cells were subcutaneously injected into nude mouse, and the specimens were harvested from the injection site for histological analysis after six-weeks injection.

Results Cell proliferation was significantly inhibited when PEDF was overexpressed. Transwell tests showed that PDEF overexpression could suppress the migration and invasion of SW620 cells. In contrast, the ability of proliferation, migration and invasion became stronger when PDEF was knocked down. Further flow cytometry showed that overexpression of PDEF could reduce the ratio of cells at the $\mathrm{G} 2 / \mathrm{M}$ phase. In addition, subcutaneous transplanted tumors overexpressing PDEF in the xenograft model were significantly smaller than the control group.
Conclusions Overexpression of PDEF could inhibit the proliferation and invasion of CRC cell in vitro and in vivo. Our study suggested that PDEF could serve as a potential tumor biomarker and drug target in CRC.

\section{IDDF2019-ABS-0270 ECT2 INCREASES EGFR EXPRESSION AND TUMORIGENICITY BY PROMOTING CDC42 ACTIVITY IN PANCREATIC CANCER}

Junxiong Wang*. Department of Gastroenterology, Beijing Friendship Hospital, Capital Medical University, China

\subsection{6/gutjnl-2019-IDDFabstracts.54}

Background The poor prognosis of patients with pancreatic ductal adenocarcinoma (PDAC) is partially attributed to the invasive and metastatic behavior of this disease. Epithelial cell transforming 2 (ECT2) is a guanine nucleotide exchange factor (GEF) of the Rho family of GTPases. It has also been reported that upregulation of ECT2 in pancreatic cancer, but the role and mechanism of ECT2 have not been previously determined. Methods Expression of ECT2 in PDAC and PDAC cell lines was characterized using immunohistochemistry and Western blotting. ECT2 was overexpressed via retroviral transduction and knocked down in PDAC cells using shRNA. Cell proliferation was determined using a colorimetric cell viability assay, and cell migration and invasion using transwells. Expression of markers of epithelial-mesenchyme transition (EMT) was assayed by Western blotting. ECT2 and cell division cycle 42 (Cdc42) were interrogated by immunoprecipitation and Western blot. The functional role of Cdc42 was determined using siRNA.

Results We found ECT2 was significantly upregulated in PDAC tissues and cells, correlated with more advanced clinicopathological features. ECT2 regulated invasion and migration of PDAC cells in vitro and in vivo. ECT2 silencing downregulated EGFR expression via accelerating EGFR degradation in pancreatic cancer cells. Immunoprecipitation assay further confirmed that ECT2 interacted with Cdc42. More importantly, ECT2 silence markedly decreases Cdc42 activity. The promoting effect of ECT2 on migration can be decreased by the siRNA against Cdc42.

Conclusions Overall, this study shows that ECT2 increases EGFR and promotes tumorigenicity of pancreatic cancer cells by enhancing Cdc42 activity.

IDDF2019-ABS-0275
DECREASED EXPRESSION OF TLE1
PROMOTES CELL APOPTOSIS AND
INDUCES ABERRANT AUTOPHAGY IN
CROHN'S DISEASE

Chenwen Cai*, Meilan Huang, Lijie Lai, Yuqi Qiao, Qing Zheng, Jun Shen, Zhihua Ran. Division of Gastroenterology and Hepatology, Renji Hospital, School of Medicine, Shanghai Jiao Tong University; Shanghai Institute of Digestive Disease; Shanghai Inflammatory Bowel Disease Research Center, China

\subsection{6/gutjnl-2019-IDDFabstracts.55}

Background Transducin-like enhancer of split 1 (TLE1) has been reported as co-repressor of multiple transcriptional factors. The association between TLE1 and the pathogenesis of 